\title{
DNA-guided genome editing tool
}

\author{
Dandan Zhang \& Jian-Feng $\mathrm{Li}^{*}$ \\ Key Laboratory of Gene Engineering of the Ministry of Education, State Key Laboratory of Biocontrol, Guangdong Provincial \\ Key Laboratory of Plant Resources, School of Life Sciences, Sun Yat-sen University, Guangzhou 510275, China \\ Received May 12, 2016; accepted May 14, 2016; published online May 19, 2016
}

Citation: $\quad$ Zhang, D., and Li, J.F. (2016). DNA-guided genome editing tool. Sci China Life Sci 59, 740-741. doi: 10.1007/s11427-016-0128-8

Technologies for targeted modification of eukaryotic genomes are of great value for elucidating and manipulating gene functions in fundamental and applied biological research. In the past two decades, genome editing tools have evolved quickly from random DNA mutagenesis-inducing chemicals or ionizing radiation to programmable sequencespecific nucleases (SSNs) with the CRISPR/Cas technology as the most significant breakthrough (Gaj et al., 2013). When the CRISPR/Cas system is still gaining snowballing popularity, a recent study from Gao and colleagues published in Nature Biotechnology has made another big splash in the field of genome editing by identifying the archaeal Argonaute protein as a novel type of SSN rivaling the CRISPR/Cas technology for genome editing in human cells (Gao et al., 2016). By opening up a new avenue for genome editing in eukaryotes, this work will undoubtedly pose a profound impact on many aspects of biological research.

Introducing a frameshift mutation or sequence alteration at an intended genomic locus leading to gene inactivation or functional modification is a common strategy for studying unknown gene functions or retuning gene activities related to human diseases or agricultural traits. In fact, gene mutagenesis can occur spontaneously during DNA repair after a DNA double-strand break is precisely created at the desired site within the target gene, which is what SSNs have been designed to fulfill. Homing endonucleases, zinc finger nucleases (ZFNs) and transcription activator-like effector nucleases (TALENs) represent earlier generations of SSNs, whose DNA recognition is achieved through DNA-binding domains inside the nuclease proteins. Consequently, repro-

*Corresponding author (email: lijfeng3@mail.sysu.edu.cn) gramming new DNA targeting specificity involves sophisticated design and demanding reconstruction of the DNAbinding protein domains. These weaknesses also dramatically limit the multiplexability of this type of SSNs. It is therefore not surprising that the CRISPR/Cas technology, which utilizes a synthetic guide RNA (sgRNA) to ferry the Streptococcus pyogenes Cas9 (SpCas9) nuclease to the genomic target through RNA-DNA complementarity, could readily exceed ZFNs and TALENs due to its unprecedented simplicity and multiplexability (Doudna and Charpentier, 2014). Even so, the CRISPR/Cas technology is blemished with frequent off-target effects, particularly in mammalian cells. The obligatory requirement of a protospacer adjacent motif (PAM) in the target sequence and the poor editing efficiencies of SpCas9 at targets with extremely low or high GC contents also limit the selection of accessible targets in a given genome.

Argonaute proteins are an ancient family of endonucleases that play essential roles in host antiviral defense and gene regulation. Eukaryotic Argonautes are guided by small single-stranded RNA such as siRNA and miRNA to cleave single-stranded RNA targets including endogenous mRNA and invading viral RNA. Interestingly, two prokaryotic homologues, namely Thermus thermophilus Argonaute (TtAgo) and Pyrococcus furiosus Argonaute (PfAgo), were found to utilize small $5^{\prime}$-phosphorylated single-stranded DNA (ssDNA) as guides to cleave double-stranded DNA targets within trespassing plasmids (Swarts et al., 2014; Swarts et al., 2015). Although these pioneering findings may suggest the possibility of developing DNA-guided genome editing tools, the high working temperatures $\left(\geqslant 65^{\circ} \mathrm{C}\right)$ of TtAgo and PfAgo, which are far above the physiological 
temperatures for eukaryotic organisms, make them unsuitable for eukaryotic genome editing.

The work by Gao and co-workers now breaks the high temperature constraint of DNA-guided Argonaute nucleases by discovering that the Natronobacterium gregoryi Argonaute (NgAgo) was able to cleave target plasmid DNA at $37^{\circ} \mathrm{C}$ in both $E$. coli and human HeLa cells solely with 5 '-phosphorylated ssDNA as guides. They next extensively tested the editing of eight different endogenous genes with a total of 47 ssDNA guides in several human and mammalian cell lines and obtained efficient editing with all guides. In particular, the authors demonstrated that the ssDNA-guided NgAgo and the sgRNA-guided SpCas9 could lead to comparable efficiencies in editing the same plasmid DNA or endogenous DYRK1A gene in HeLa cells. Notably, they found that NgAgo performed substantially better than SpCas9 when aiming at some GC-rich loci within target genes. The authors also clarified that the optimal length of the ssDNA guide for NgAgo is $24 \mathrm{nt}$ and any single mismatch between the guide and the target DNA could dramatically compromise the editing efficiencies. They further accomplished homology recombination-mediated DNA insertion at the DYRK1A gene in human 293T cells with the $\mathrm{NgAgo}$ system, and found that only SpCas9 but not NgAgo led to off-target DNA insertion.

Evidence presented in this work suggests that the $\mathrm{NgAgo}$ system may carry a few striking advantages over the SpCas9 system. First, NgAgo employs a longer guide sequence (24 nt) and exhibits a lower tolerance to guide-target mismatches than SpCas9, thus ensuring a higher editing specificity; second, $\mathrm{NgAgo}$ does not impose any PAM requirement to the target sequence and is highly tolerant to GC-rich target sequences, thus offering a much broader range of target selection than SpCas9; last but not least, the gene size of $\mathrm{NgAgo}(\sim 2.7 \mathrm{~kb})$ is significantly smaller than that of SpCas9 $(\sim 4.2 \mathrm{~kb})$ and SaCas9 $(\sim 3.3 \mathrm{~kb}$, the smallest Cas9 known so far), and 5'-phosphorylated ssDNA guides are much cheaper for commercial synthesis and more stable than the sgRNA guides, together making the NgAgo system more amenable to work with than the CRISPR/Cas system during cloning/synthesis and cell delivery.

It is foreseeable that follow-up exploration will involve the co-delivery of NgAgo-encoding plasmids and commercially synthesized 5'-phosphorylated ssDNA into mammalian zygotes via microinjection to generate genome-edited whole animals. To enable the $\mathrm{NgAgo}$ system to work for other important eukaryotic model organisms including plants, fruitfly, nematode and zebrafish, improvement will be needed to reduce its working temperature down to the range of room temperature. Accordingly, it will be necessary to carefully confirm the absence of endogenous small 5 '-phosphorylated ssDNA in these organisms that could mislead NgAgo to off-target sites. Although preliminary data by Gao and colleagues suggested that $\mathrm{NgAgo}$ is highly specific in mammalian cells (Gao et al., 2016), systematic evaluation of its off-target effects with unbiased wholegenome sequencing will be indispensable. In addition, the multiplexability of the NgAgo system still awaits evaluation. On the other hand, addressing the fundamental questions regarding the action mechanisms of $\mathrm{NgAgo-based}$ immune system in $N$. gregoryi will be needed, which may in turn guide the improvement of the $\mathrm{NgAgo}$ system. For instance, understanding the mechanism of how archaea generate small 5'-phosphorylated ssDNA may inspire us to find ways to produce customized ssDNA guides in vivo in eukaryotic cells; knowledge about the structural basis of NgAgo-ssDNA and NgAgo-target DNA interactions may guide the engineering of $\mathrm{NgAgo}$ variants allowing ssDNA exchange in vivo or adopting lower reaction temperatures. Although it is uncertain whether the $\mathrm{NgAgo}$ system will eventually replace the prevailing CRISPR/Cas system or be merely an attractive complement to the latter, there is every reason to believe that future efforts investing on this groundbreaking technology will pay huge dividend to basic and applied biological research.

Compliance and ethics The author(s) declare that they have no conflict of interest.

Doudna, J.A., and Charpentier, E. (2014) Genome editing: the new frontier of genome engineering with CRISPR-Cas9. Science 346, 1258096

Gaj, T., Gersbach, C.A., and Barbas III, C.F. (2013) ZFN, TALEN, and CRISPR/Cas-based methods for genome engineering. Trends Biotechnol 31, 397-405

Gao, F., Shen, X.Z., Jiang, F., Wu, Y., and Han, C. (2016) DNA-guided genome editing using the Natronobacterium gregoryi Argonaute. Nat Biotechnol doi: 10.1038/nbt.3547

Swarts, D.C., Hegge, J.W., Hinojo, I., Shiimori, M., Ellis, M.A., Dumrongkulraksa, J., Terns, R.M., Terns, M.P., and van der Oost, J. (2015) Argonaute of the archaeon Pyrococcus furiosus is a DNA-guided nuclease that targets cognate DNA. Nucleic acids Res 43, 5120-5129

Swarts, D.C., Jore, M.M., Westra, E.R., Zhu, Y., Janssen, J.H., Snijders, A.P., Wang, Y., Patel, D.J., Berenguer, J., Brouns, S.J.J., and van der Oost, J. (2014) DNA-guided DNA interference by a prokaryotic Argonaute. Nature 507, 258-261

Open Access This article is distributed under the terms of the Creative Commons Attribution License which permits any use, distribution, and reproduction in any medium, provided the original author(s) and source are credited. 